\title{
Islamic Copper-Based Metal Artefacts from the Garb Al-Andalus. A Multidisciplinary Approach on the Alcáçova of Mārtulah (Mértola, South of Portugal)
}

Carlo Emanuele Bottaini Universidade de Évora https://orcid.org/0000-0003-2464-468X

\section{Susana Gómez-Martínez}

Universidade de Evora

Rui Bordalo

Universidade Catolica Portuguesa

Massimo Beltrame

Universidade de Evora

José Mirão

Universidade de Evora

Lígia Rafael

Municipality of the city of Mertola

Nick Schiavon ( $\square$ schiavon@uevora.pt)

Universidade de Evora https://orcid.org/0000-0002-9744-0808

\section{Research Article}

Keywords: Islamic metallurgy, Mértola, al-Andalus, EDXRF, SEM/EDS

Posted Date: December 29th, 2021

DOI: https://doi.org/10.21203/rs.3.rs-1172830/v1

License: (a) (i) This work is licensed under a Creative Commons Attribution 4.0 International License. Read Full License 


\section{Abstract}

A multidisciplinary approach has been applied to investigate the production technology of a collection of copper-based artefacts found during archaeological excavation campaigns carried out in the Almohad neighbourhood of Mārtulah, the Islamic name of modern Mértola (South of Portugal). In stark contrast to other Islamic materials found in the same site such as common and finely decorated pottery, glass, and bone artefacts, metal objects have received less attention despite the number of artefacts recovered.

This study focuses on the chemical characterisation of 172 copper-based artefacts dating back to the 12th and the first half of the 13th centuries. The artefacts are daily use objects and consist of personal ornaments (earrings, rings, and casket ornaments), tools (spindles, spatulas, and oil lamp sticks) and artefacts with unknown functions. X-ray fluorescence Spectroscopy (XRF) and Scanning Electron Microscopy coupled with Energy Dispersive X-ray Spectroscopy (SEM/EDS), provided information not only about technological issues, as well as on the socio-economic implications of metal consumption at Islamic Mértola. The results revealed that metals were produced with a variety of Cu-based alloys, namely unalloyed copper, brasses, bronzes, and ternary alloys, by mixing $\mathrm{Cu}, \mathrm{Zn}$ and $\mathrm{Sn}$ and $\mathrm{Pb}$ without any apparent consistency, as a likely result of recurrent recycling and mixing scrap metals practices or use of minerals available locally.

\section{Introduction}

Islamic culture provided a very important contribution to European history and modern science and technology [1, 2]. In fact, since the 7th century AD, scholars from the Muslim world stand out in virtually every field of knowledge, being at the forefront of scientific advance and technological innovation in a wide range of research fields such as astronomy, medicine, mathematics, cartography, and agriculture [3, 4].

Since the arrival of the Muslim army led by Tariq ibn Ziyad (711 AD), who firstly crossed the Strait of Gibraltar from the North African coast, and up to the end of the 13th century, when the Christian Reconquest was completed in the west of al-Andalus, the Iberian Peninsula was gradually and actively involved in this climate of cultural and scientific development, generally known as Islamic Golden Age [5, 6]. Along this period of prosperity that lasted about five centuries, the Iberian Peninsula also played a central role as one of the major points of transmission of Islamic culture and technology to the rest of Europe.

Towns in al-Andalus like Seville, Cordoba or Granada progressively became the centre of social life and political power, also establishing themselves as commercial hubs where manufacturing activities (e.g., pottery, metalwork, tannery) and different forms of art (e.g., textiles, illuminated manuscripts, woodwork, architecture, ceramics, and metalwork) flourished. Within this scenario, Mārtulah, located at the confluence between the Guadiana River and the Oeiras creek, benefited from its strategic location as the 
last navigable point of the river, functioning as a commercial hub able to link the Atlantic Ocean with Northern Africa, al-Andalus and the Mediterranean Sea [7, 8] (Fig. 1).

The 12th and 13th centuries, i.e., the period when the metal artefacts analysed in this paper were produced and used, was characterized by a great trade development despite a political instability caused both by internal crises within the Islamic community and by increasing external pressure caused by the advancing Reconquest by the Christian Iberian kingdoms. From the beginning of the 2nd millennium, periods of political fragmentation (with the creation of the so-called Taifas), alternated with periods of reunification, especially under the Almoravid and Almohad dynasties. The conquest of Mértola by the Portuguese king Sancho II in 1238 was a key moment in the southward advance of the Christian forces that, in 1249, with King Afonso III, put an end to the Islamic presence in southern Portugal by conquering the Algarve region.

The systematic archaeological excavations carried out along more than 40 years in the urban area of the modern Mértola have shed light on this historical period, allowing to reconstruct its history and the evolution of the town since the Iron Age period and across the centuries. Important archaeological vestiges, including monumental buildings, still stand as evidence of Islamic Mārtulah. One of the most relevant excavated areas is the Almohad neighbourhood, located in the Alcazaba of Mértola, i.e., the walled fortification, in an area located on the northern slope of the castle that overlooks the town (Fig. 2).

The area where the Almohad neighbourhood is located stands on an artificial platform built on a late Roman cryptoporticus, already occupied by buildings richly decorated with mosaics during the Late Antiquity (5th -8th centuries). Around the 12th century, it was used for the edification of a neighbourhood that was abandoned soon after the Christian conquest of Mértola. In the following centuries, the area served for different purposes: it was used as a Christian cemetery until the 18th century, as a vegetable garden afterwards and from the beginning of the 20th century onwards as a football pitch. The metals analysed in this paper are from the excavated area, where 15 houses from the Islamic period have been dug [9] (Fig. 2).

Even though Islamic copper-based artefacts have been recurrently found at different excavations carried out at Mértola, this paper present for the first time the results of a large-scale analytical program aimed at characterising the chemical composition of a collection of metals composed of 172 artefacts. In fact, the lack of analytical data on Islamic metallurgy is a common trait to other Iberian regions. Very few studies have been dedicated to this topic so far, with few exceptions, i.e., Madinat al-Zahra (Córdoba) [10], Denia (Alicante) [11, 12], Silves (Faro) [13], and a collection of oil lamps from different Portuguese sites [14].

The analytical strategy adopted in this work was oriented towards the acquisition of information on the composition of copper alloys by means of portable and hand-held X-ray fluorescence spectroscopy (XRF) and scanning electron microscopy (SEM) coupled with energy dispersive X-ray spectrometry (EDS). The aim of the research was to provide an overview of the metal production technology in the site while, at the same time shedding light on social and economic issues related to the use of metal in Mértola during the last phase of Islamic rule. 


\section{Materials And Methods}

\section{Materials}

The collection of objects analysed in this paper represents a selection of the copper-based artefacts recovered to date from the Almohad neighbourhood of Mārtulah. The assemblage includes 172 artefacts that, based on their typology, can be subdivided into three different groups, namely: a) tools (i.e., spindles, oil lamp sticks, and spatulas), b) ornaments (i.e., rings, earrings, buckles, casket ornaments), and c) fragmented object of undetermined function (Fig. 3). Regardless of their typological characteristics, it is important to stress that all the artefacts analysed here come from domestic contexts and can therefore be considered as objects of daily use.

\section{Methods}

The XRF equipment used was a Bruker TRACER III-SD handheld spectrometer equipped with a rhodium anode tube and a Silicon Drift Detector with a resolution of $140 \mathrm{eV}$ at Mn Ka FWHM $5.9 \mathrm{keV}$. The operating conditions were $40 \mathrm{kV}$ and $3 \mu \mathrm{A}$ current with an $\mathrm{Al} / \mathrm{Ti}$ filter $(304.8 \mu \mathrm{m}$ aluminium/25.4 $\mu \mathrm{m}$ titanium) and $60 \mathrm{~s}$ acquisition time. The spectra were acquired using the Bruker S1PXRF v.3.8.30 software and Bruker ARTAX v.5.3.0.0 software for the first spectra evaluation. For system calibration, analyses of certified copper alloys were used, namely five standards from certified reference materials [15] and three standard reference materials (National Bureau of Standards Standard Reference Material 1107, 1110 and 1113). Quantification was performed using Bruker S1CalProcess v.2.2.33 software to find the concentration of the unknown samples.

A few selected artefacts were also investigated by SEM/EDS using a HITACHI S3700N interfaced with a QUANTAX EDS microanalysis system equipped with a Bruker AXS 5010XFlash 5010 Silicon Drift Detector (129 eV Spectral Resolution at FWHM/Mn Ka). The operating conditions for EDS analysis were set as follows: backscattered electron mode (BSEM), $20 \mathrm{kV}$ accelerating voltage, $\sim 10 \mathrm{~mm}$ working distance and $90 \mu \mathrm{A}$ emission current. Spectra were analysed using Bruker Esprit 1.9 software.

\section{Results And Discussion}

Results are summarized in Table 1. Even though we recognise the arbitrary nature of establishing a threshold values system for defining the different types of ancient alloys, to better organize the collected data, the following scheme proposed by Gaudenzi Asinelli et al. has been adopted [16]: 
Table 1

Elemental composition of the artefacts from the Almohad neighbourhood of Mārtulah (wt.\%); n.d.: not detected.

\begin{tabular}{|c|c|c|c|c|c|c|c|c|c|c|}
\hline ID & Function & $\mathrm{Cu}$ & $\mathrm{Zn}$ & Sn & $\mathrm{Pb}$ & $\mathrm{Fe}$ & As & $\mathrm{Ag}$ & $\mathrm{Ni}$ & $\mathrm{Sb}$ \\
\hline \multicolumn{11}{|l|}{ Brass } \\
\hline $\begin{array}{l}\text { AA-01- } \\
02\end{array}$ & $\begin{array}{l}\text { casket } \\
\text { ornament }\end{array}$ & 91.90 & 4.95 & 1.40 & 0.75 & 0.30 & 0.46 & 0.07 & 0.02 & 0.15 \\
\hline $\begin{array}{l}\text { AA-01- } \\
04\end{array}$ & $\begin{array}{l}\text { casket } \\
\text { ornament }\end{array}$ & 90.30 & 6.60 & 1.60 & 0.93 & 0.07 & 0.20 & 0.09 & 0.01 & 0.20 \\
\hline $\begin{array}{l}\text { AA-01- } \\
13\end{array}$ & $\begin{array}{l}\text { casket } \\
\text { ornament }\end{array}$ & 86.75 & 12.40 & 0.48 & 0.08 & 0.19 & 0.10 & n.d. & n.d. & n.d. \\
\hline $\begin{array}{l}\text { AA-01- } \\
14\end{array}$ & $\begin{array}{l}\text { casket } \\
\text { ornament }\end{array}$ & 89.35 & 8.35 & 0.90 & 0.40 & 0.35 & 0.40 & 0.09 & 0.01 & 0.15 \\
\hline $\begin{array}{l}\text { AA-01- } \\
16\end{array}$ & $\begin{array}{l}\text { casket } \\
\text { ornament }\end{array}$ & 91.00 & 7.20 & 0.33 & 0.17 & 0.30 & 0.90 & 0.06 & 0.01 & 0.03 \\
\hline $\begin{array}{l}\text { AA-01- } \\
17\end{array}$ & $\begin{array}{l}\text { casket } \\
\text { ornament }\end{array}$ & 89.30 & 8.75 & 1.20 & 0.22 & 0.02 & 0.42 & 0.09 & n.d. & n.d. \\
\hline $\begin{array}{l}\text { AA-01- } \\
20\end{array}$ & $\begin{array}{l}\text { casket } \\
\text { ornament }\end{array}$ & 88.70 & 8.45 & 0.50 & 0.90 & 0.30 & 1.15 & n.d. & n.d. & n.d. \\
\hline $\begin{array}{l}\text { AA-02- } \\
25\end{array}$ & $\begin{array}{l}\text { casket } \\
\text { ornament }\end{array}$ & 86.45 & 10.40 & 0.60 & 0.95 & 0.32 & 0.80 & 0.48 & n.d. & n.d. \\
\hline $\begin{array}{l}\text { AA-02- } \\
26\end{array}$ & $\begin{array}{l}\text { casket } \\
\text { ornament }\end{array}$ & 86.91 & 10.34 & 0.91 & 1.15 & 0.22 & 0.15 & 0.32 & n.d. & n.d. \\
\hline $\begin{array}{l}\text { AA-02- } \\
27\end{array}$ & $\begin{array}{l}\text { casket } \\
\text { ornament }\end{array}$ & 88.12 & 7.90 & 1.40 & 1.55 & 0.25 & 0.23 & 0.26 & 0.02 & 0.27 \\
\hline $\begin{array}{l}\text { AA-02- } \\
28\end{array}$ & $\begin{array}{l}\text { casket } \\
\text { ornament }\end{array}$ & 93.05 & 4.62 & 0.70 & 0.75 & 0.29 & 0.15 & 0.29 & n.d. & 0.15 \\
\hline $\begin{array}{l}\text { AA-02- } \\
29\end{array}$ & $\begin{array}{l}\text { casket } \\
\text { ornament }\end{array}$ & 77.31 & 22.37 & n.d. & 0.10 & 0.02 & 0.13 & n.d. & 0.07 & n.d. \\
\hline $\begin{array}{l}\text { AA-02- } \\
30\end{array}$ & $\begin{array}{l}\text { casket } \\
\text { ornament }\end{array}$ & 83.92 & 14.52 & n.d. & 1.10 & 0.26 & 0.20 & n.d. & n.d. & n.d. \\
\hline $\begin{array}{l}\text { AA-02- } \\
32\end{array}$ & $\begin{array}{l}\text { casket } \\
\text { ornament }\end{array}$ & 83.56 & 14.74 & 1.10 & 0.15 & 0.23 & 0.20 & n.d. & 0.02 & n.d. \\
\hline $\begin{array}{l}\text { AA-02- } \\
33\end{array}$ & $\begin{array}{l}\text { casket } \\
\text { ornament }\end{array}$ & 78.31 & 20.30 & 1.10 & 0.05 & 0.03 & 0.12 & 0.06 & 0.03 & n.d. \\
\hline $\begin{array}{l}\text { AA-02- } \\
36\end{array}$ & $\begin{array}{l}\text { casket } \\
\text { ornament }\end{array}$ & 86.15 & 12.07 & 1.25 & 0.30 & 0.10 & 0.13 & n.d. & n.d. & n.d. \\
\hline $\begin{array}{l}\text { AA-02- } \\
37\end{array}$ & $\begin{array}{l}\text { casket } \\
\text { ornament }\end{array}$ & 86.55 & 10.20 & 1.70 & 1.02 & 0.10 & 0.19 & 0.07 & 0.02 & 0.15 \\
\hline
\end{tabular}




\begin{tabular}{|c|c|c|c|c|c|c|c|c|c|c|}
\hline ID & Function & $\mathrm{Cu}$ & $\mathrm{Zn}$ & Sn & $\mathrm{Pb}$ & $\mathrm{Fe}$ & As & $\mathrm{Ag}$ & $\mathrm{Ni}$ & $S b$ \\
\hline $\begin{array}{l}\text { AA-02- } \\
46\end{array}$ & $\begin{array}{l}\text { casket } \\
\text { ornament }\end{array}$ & 90.46 & 6.52 & 1.37 & 0.95 & 0.20 & 0.24 & 0.07 & 0.02 & 0.17 \\
\hline $\begin{array}{l}\text { AA-02- } \\
54\end{array}$ & $\begin{array}{l}\text { casket } \\
\text { ornament }\end{array}$ & 88.80 & 9.95 & n.d. & 0.15 & 0.08 & 0.70 & 0.11 & 0.17 & 0.04 \\
\hline $\begin{array}{l}\text { AA-03- } \\
62\end{array}$ & $\begin{array}{l}\text { casket } \\
\text { ornament }\end{array}$ & 81.93 & 14.11 & 1.40 & 1.35 & 0.11 & 0.90 & 0.20 & n.d. & n.d. \\
\hline $\begin{array}{l}\text { AN-01- } \\
36\end{array}$ & ring & 87.51 & 10.50 & 0.40 & 0.55 & 0.27 & 0.70 & 0.07 & n.d. & n.d. \\
\hline $\begin{array}{l}\text { AR-01- } \\
35\end{array}$ & ring & 85.22 & 12.07 & 1.38 & 0.80 & 0.26 & 0.22 & n.d. & 0.05 & n.d. \\
\hline $\begin{array}{l}\text { BC-01- } \\
05\end{array}$ & earring & 94.32 & 2.47 & 1.16 & 1.40 & 0.10 & 0.55 & n.d. & n.d. & n.d. \\
\hline $\begin{array}{l}\text { BC-01- } \\
06\end{array}$ & earring & 91.04 & 7.07 & 0.70 & 0.40 & 0.05 & 0.66 & 0.08 & n.d. & n.d. \\
\hline $\begin{array}{l}\text { BC-01- } \\
07\end{array}$ & earring & 93.41 & 4.75 & 0.65 & 0.60 & 0.06 & 0.15 & 0.14 & 0.01 & 0.23 \\
\hline $\begin{array}{l}\text { BC-01- } \\
11\end{array}$ & earring & 89.79 & 7.98 & 1.35 & 0.21 & 0.03 & 0.55 & 0.09 & n.d. & n.d. \\
\hline $\begin{array}{l}\text { BC-01- } \\
16\end{array}$ & earring & 88.53 & 9.41 & 1.05 & 0.45 & 0.10 & 0.31 & 0.15 & n.d. & n.d. \\
\hline $\begin{array}{l}\text { BC-01- } \\
17\end{array}$ & earring & 82.88 & 14.12 & 1.35 & 0.65 & 0.20 & 0.80 & n.d. & n.d. & n.d. \\
\hline $\begin{array}{l}\text { BC-01- } \\
18\end{array}$ & earring & 85.47 & 13.08 & 0.85 & 0.15 & 0.07 & 0.25 & 0.13 & n.d. & n.d. \\
\hline $\begin{array}{l}\text { BC-01- } \\
19\end{array}$ & earring & 88.67 & 9.23 & 0.65 & 0.22 & 0.06 & 1.10 & 0.07 & n.d. & n.d. \\
\hline $\begin{array}{l}\text { BC-01- } \\
21\end{array}$ & earring & 82.98 & 14.72 & 1.20 & 0.25 & 0.05 & 0.80 & n.d. & n.d. & n.d. \\
\hline $\begin{array}{l}\text { BC-01- } \\
26\end{array}$ & earring & 86.96 & 11.55 & 0.75 & 0.10 & 0.06 & 0.50 & 0.08 & n.d. & n.d. \\
\hline $\begin{array}{l}\text { BC-01- } \\
30\end{array}$ & earring & 92.38 & 4.98 & 1.16 & 0.95 & 0.12 & 0.40 & n.d. & 0.01 & n.d. \\
\hline $\begin{array}{l}\text { BC-01- } \\
42\end{array}$ & earring & 88.27 & 8.71 & 0.90 & 0.48 & 0.14 & 1.50 & n.d. & n.d. & n.d. \\
\hline $\begin{array}{l}\text { BC-01- } \\
46\end{array}$ & earring & 91.79 & 5.76 & 0.88 & 0.62 & 0.10 & 0.85 & n.d. & n.d. & n.d. \\
\hline $\begin{array}{l}\text { BC-01- } \\
55\end{array}$ & earring & 88.69 & 7.73 & 1.85 & 0.85 & 0.20 & 0.40 & n.d. & 0.02 & 0.26 \\
\hline
\end{tabular}




\begin{tabular}{|c|c|c|c|c|c|c|c|c|c|c|}
\hline ID & Function & $\mathrm{Cu}$ & $\mathrm{Zn}$ & Sn & $\mathrm{Pb}$ & $\mathrm{Fe}$ & As & $\mathrm{Ag}$ & $\mathrm{Ni}$ & Sb \\
\hline $\begin{array}{l}\text { BC-01- } \\
61\end{array}$ & earring & 90.92 & 5.75 & 1.35 & 1.00 & 0.26 & 0.35 & 0.11 & 0.02 & 0.24 \\
\hline $\begin{array}{l}\text { BC-01- } \\
62\end{array}$ & earring & 87.33 & 10.32 & 1.15 & 0.21 & 0.03 & 0.90 & 0.06 & n.d. & n.d. \\
\hline $\begin{array}{l}\text { BC-01- } \\
66\end{array}$ & earring & 90.56 & 5.61 & 1.80 & 0.95 & 0.25 & 0.55 & 0.10 & n.d. & 0.18 \\
\hline $\begin{array}{l}\text { BC-01- } \\
72\end{array}$ & earring & 86.07 & 11.43 & 1.30 & 0.50 & 0.02 & 0.60 & 0.07 & 0.01 & n.d. \\
\hline $\begin{array}{l}\text { BC-01- } \\
76\end{array}$ & earring & 91.58 & 6.44 & 0.75 & 0.60 & 0.04 & 0.25 & 0.15 & 0.01 & 0.18 \\
\hline $\begin{array}{l}\text { BC-01- } \\
89\end{array}$ & earring & 84.81 & 12.14 & 1.63 & 0.68 & 0.06 & 0.35 & 0.09 & 0.01 & 0.23 \\
\hline $\begin{array}{l}\text { BC-03- } \\
101\end{array}$ & earring & 83.48 & 14.74 & 1.55 & 0.05 & 0.03 & 0.15 & n.d. & n.d. & n.d. \\
\hline $\begin{array}{l}\text { BC-03- } \\
105\end{array}$ & earring & 88.35 & 9.00 & 1.25 & 0.60 & 0.20 & 0.30 & 0.09 & 0.01 & 0.20 \\
\hline $\begin{array}{l}\text { BC-03- } \\
106\end{array}$ & earring & 87.23 & 9.42 & 1.70 & 1.05 & 0.05 & 0.30 & 0.07 & 0.01 & 0.17 \\
\hline $\begin{array}{l}\text { BC-03- } \\
108\end{array}$ & earring & 83.68 & 14.32 & 0.87 & 0.38 & 0.10 & 0.65 & n.d. & n.d. & n.d. \\
\hline $\begin{array}{l}\text { BC-03- } \\
109\end{array}$ & earring & 88.55 & 8.19 & 1.15 & 1.40 & 0.25 & 0.30 & 0.16 & n.d. & n.d. \\
\hline $\begin{array}{l}\text { BC-03- } \\
115\end{array}$ & earring & 90.60 & 7.60 & 0.48 & 0.60 & 0.13 & 0.33 & 0.08 & 0.01 & 0.17 \\
\hline $\begin{array}{l}\text { BC-03- } \\
97\end{array}$ & earring & 91.97 & 5.10 & 1.30 & 0.65 & 0.19 & 0.70 & 0.09 & n.d. & n.d. \\
\hline $\begin{array}{l}\text { BC-03- } \\
98\end{array}$ & earring & 83.50 & 15.10 & 1.17 & 0.05 & 0.04 & 0.08 & n.d. & 0.06 & n.d. \\
\hline $\begin{array}{l}\text { DV-02- } \\
44\end{array}$ & undetermined & 93.28 & 4.19 & 0.90 & 0.25 & 0.21 & 0.74 & 0.43 & n.d. & n.d. \\
\hline $\begin{array}{l}\text { DV-02- } \\
75\end{array}$ & undetermined & 89.80 & 7.65 & 0.95 & 0.85 & 0.09 & 0.47 & 0.06 & 0.01 & 0.12 \\
\hline $\begin{array}{l}\text { DV-02- } \\
80\end{array}$ & undetermined & 89.87 & 6.45 & 1.30 & 1.93 & 0.12 & 0.22 & n.d. & 0.01 & 0.10 \\
\hline $\begin{array}{l}\text { DV-03- } \\
113\end{array}$ & sword sheath & 93.98 & 2.27 & 0.80 & 1.35 & 0.18 & 0.60 & 0.16 & 0.07 & 0.59 \\
\hline $\begin{array}{l}\text { DV-03- } \\
86\end{array}$ & undetermined & 88.30 & 8.90 & 1.55 & 0.48 & 0.03 & 0.48 & 0.08 & 0.02 & 0.16 \\
\hline
\end{tabular}




\begin{tabular}{|c|c|c|c|c|c|c|c|c|c|c|}
\hline ID & Function & $\mathrm{Cu}$ & $\mathrm{Zn}$ & Sn & $\mathrm{Pb}$ & $\mathrm{Fe}$ & As & $\mathrm{Ag}$ & $\mathrm{Ni}$ & $S b$ \\
\hline $\begin{array}{l}\text { DV-03- } \\
89\end{array}$ & undetermined & 89.46 & 8.10 & 0.95 & 0.47 & 0.35 & 0.45 & 0.07 & 0.02 & 0.13 \\
\hline $\begin{array}{l}\text { EC-01- } \\
05\end{array}$ & oil lamp stick & 87.86 & 11.65 & n.d. & 0.30 & 0.07 & 0.12 & n.d. & n.d. & n.d. \\
\hline $\begin{array}{l}\text { EC-01- } \\
07\end{array}$ & oil lamp stick & 85.70 & 14.05 & n.d. & 0.10 & 0.07 & 0.03 & 0.05 & n.d. & n.d. \\
\hline $\begin{array}{l}\text { EC-01- } \\
09\end{array}$ & oil lamp stick & 89.74 & 6.94 & 1.65 & 1.15 & 0.25 & 0.21 & 0.06 & n.d. & n.d. \\
\hline $\begin{array}{l}\text { EC-01- } \\
12\end{array}$ & oil lamp stick & 85.54 & 12.43 & 0.10 & 1.07 & 0.20 & 0.47 & 0.14 & 0.05 & n.d. \\
\hline $\begin{array}{l}\text { EC-01- } \\
15\end{array}$ & oil lamp stick & 88.13 & 10.67 & n.d. & 0.55 & 0.03 & 0.10 & 0.21 & 0.31 & n.d. \\
\hline $\begin{array}{l}\text { EC-01- } \\
16\end{array}$ & oil lamp stick & 88.89 & 8.70 & 0.60 & 0.38 & 0.35 & 0.78 & 0.14 & 0.01 & 0.15 \\
\hline $\begin{array}{l}\text { ES-01- } \\
01\end{array}$ & spatula & 85.69 & 13.70 & 0.01 & 0.50 & 0.03 & 0.07 & n.d. & n.d. & n.d. \\
\hline $\begin{array}{l}\text { ES-01- } \\
06\end{array}$ & spatula & 92.77 & 5.18 & 0.30 & 0.54 & 0.14 & 0.90 & n.d. & 0.02 & 0.15 \\
\hline $\begin{array}{l}\text { ES-01- } \\
08\end{array}$ & spatula & 91.43 & 4.77 & 1.38 & 0.95 & 0.87 & 0.31 & 0.26 & 0.03 & n.d. \\
\hline $\begin{array}{l}\text { ES-01- } \\
11\end{array}$ & spatula & 94.88 & 2.52 & 0.49 & 0.43 & 0.16 & 1.30 & n.d. & 0.01 & 0.21 \\
\hline $\begin{array}{l}\text { ES-01- } \\
12\end{array}$ & spatula & 87.94 & 7.33 & 2.02 & 1.55 & 0.21 & 0.58 & n.d. & 0.03 & 0.34 \\
\hline $\begin{array}{l}\text { ES-01- } \\
20\end{array}$ & spatula & 89.03 & 8.07 & 0.85 & 0.77 & 0.20 & 0.71 & 0.14 & 0.05 & 0.18 \\
\hline $\begin{array}{l}\text { ES-01- } \\
22\end{array}$ & spatula & 88.14 & 7.68 & 1.55 & 1.70 & 0.15 & 0.44 & 0.12 & n.d. & 0.22 \\
\hline $\begin{array}{l}\text { ES-02- } \\
23\end{array}$ & spatula & 88.66 & 9.67 & 0.50 & 0.51 & 0.04 & 0.62 & n.d. & n.d. & n.d. \\
\hline $\begin{array}{l}\text { ES-02- } \\
27\end{array}$ & spatula & 89.26 & 8.41 & 0.74 & 0.28 & 0.10 & 1.20 & n.d. & 0.01 & n.d. \\
\hline $\begin{array}{l}\text { ES-02- } \\
29\end{array}$ & spatula & 85.99 & 10.63 & 1.40 & 1.10 & 0.15 & 0.40 & n.d. & 0.03 & 0.30 \\
\hline $\begin{array}{l}\text { ES-02- } \\
30\end{array}$ & spatula & 88.77 & 7.63 & 0.83 & 1.80 & 0.12 & 0.40 & 0.20 & 0.03 & 0.22 \\
\hline $\begin{array}{l}\text { ES-02- } \\
54\end{array}$ & spatula & 93.94 & 4.49 & 0.24 & 0.61 & 0.21 & 0.30 & 0.20 & 0.01 & n.d. \\
\hline
\end{tabular}




\begin{tabular}{|c|c|c|c|c|c|c|c|c|c|c|}
\hline ID & Function & $\mathrm{Cu}$ & $\mathrm{Zn}$ & Sn & $\mathrm{Pb}$ & $\mathrm{Fe}$ & As & $\mathrm{Ag}$ & $\mathrm{Ni}$ & $S b$ \\
\hline $\begin{array}{l}\text { PF-01- } \\
02\end{array}$ & spindle & 88.39 & 7.61 & 1.41 & 0.90 & 0.06 & 1.35 & 0.27 & 0.01 & n.d. \\
\hline $\begin{array}{l}\text { PF-01- } \\
04\end{array}$ & spindle & 90.93 & 5.08 & 2.03 & 0.49 & 0.15 & 0.50 & 0.60 & 0.01 & 0.21 \\
\hline $\begin{array}{l}\text { PF-01- } \\
06\end{array}$ & spindle & 90.36 & 7.90 & 0.38 & 0.62 & 0.20 & 0.14 & 0.15 & 0.04 & 0.21 \\
\hline $\begin{array}{l}\text { PF-01- } \\
100\end{array}$ & spindle & 93.10 & 3.67 & 1.55 & 0.52 & 0.35 & 0.23 & 0.27 & 0.01 & 0.30 \\
\hline $\begin{array}{l}\text { PF-01- } \\
104\end{array}$ & spindle & 89.45 & 8.20 & 0.61 & 0.66 & 0.40 & 0.40 & 0.11 & 0.01 & 0.16 \\
\hline $\begin{array}{l}\text { PF-01- } \\
106\end{array}$ & spindle & 84.99 & 12.06 & 0.85 & 0.88 & 0.16 & 1.05 & n.d. & 0.01 & n.d. \\
\hline $\begin{array}{l}\text { PF-01- } \\
107\end{array}$ & spindle & 85.23 & 14.08 & 0.03 & 0.32 & 0.13 & 0.14 & 0.07 & n.d. & n.d. \\
\hline $\begin{array}{l}\text { PF-01- } \\
16\end{array}$ & spindle & 88.66 & 9.23 & 1.03 & 0.13 & 0.15 & 0.80 & n.d. & n.d. & n.d. \\
\hline $\begin{array}{l}\text { PF-01- } \\
20\end{array}$ & spindle & 85.81 & 11.58 & 1.42 & 0.32 & 0.07 & 0.80 & n.d. & n.d. & n.d. \\
\hline $\begin{array}{l}\text { PF-01- } \\
27\end{array}$ & spindle & 88.54 & 9.28 & 0.40 & 0.65 & 0.13 & 0.86 & 0.14 & n.d. & n.d. \\
\hline $\begin{array}{l}\text { PF-01- } \\
32\end{array}$ & spindle & 80.91 & 15.48 & 1.90 & 0.40 & 0.16 & 1.15 & n.d. & n.d. & n.d. \\
\hline $\begin{array}{l}\text { PF-01- } \\
33\end{array}$ & spindle & 91.05 & 6.67 & 0.60 & 0.20 & 0.05 & 1.35 & 0.08 & n.d. & n.d. \\
\hline $\begin{array}{l}\text { PF-01- } \\
36\end{array}$ & spindle & 81.16 & 15.78 & 1.66 & 0.20 & 0.23 & 0.83 & 0.13 & 0.01 & n.d. \\
\hline $\begin{array}{l}\text { PF-01- } \\
37\end{array}$ & spindle & 83.62 & 13.83 & 1.00 & 0.40 & 0.25 & 0.79 & 0.11 & n.d. & n.d. \\
\hline $\begin{array}{l}\text { PF-01- } \\
47\end{array}$ & spindle & 93.09 & 4.90 & 0.70 & 0.79 & 0.28 & 0.11 & 0.13 & n.d. & n.d. \\
\hline $\begin{array}{l}\text { PF-01- } \\
54\end{array}$ & spindle & 83.75 & 14.87 & 0.29 & 0.17 & 0.07 & 0.78 & n.d. & n.d. & 0.07 \\
\hline $\begin{array}{l}\text { PF-01- } \\
65\end{array}$ & spindle & 87.95 & 9.26 & 1.20 & 0.32 & 0.04 & 1.15 & 0.07 & 0.01 & n.d. \\
\hline $\begin{array}{l}\text { PF-01- } \\
77\end{array}$ & spindle & 84.43 & 11.90 & 1.45 & 1.70 & 0.18 & 0.30 & n.d. & 0.04 & n.d. \\
\hline $\begin{array}{l}\text { PF-01- } \\
81\end{array}$ & spindle & 92.73 & 4.39 & 1.15 & 0.85 & 0.19 & 0.55 & 0.14 & n.d. & n.d. \\
\hline
\end{tabular}




\begin{tabular}{|c|c|c|c|c|c|c|c|c|c|c|}
\hline ID & Function & $\mathrm{Cu}$ & $\mathrm{Zn}$ & Sn & $\mathrm{Pb}$ & $\mathrm{Fe}$ & As & $\mathrm{Ag}$ & $\mathrm{Ni}$ & $S b$ \\
\hline $\begin{array}{l}\text { PF-01- } \\
85\end{array}$ & spindle & 89.42 & 6.98 & 0.83 & 0.88 & 0.58 & 1.20 & 0.10 & 0.01 & n.d. \\
\hline $\begin{array}{l}\text { PF-02- } \\
114\end{array}$ & spindle & 94.92 & 2.68 & 0.56 & 1.50 & 0.09 & 0.25 & n.d. & n.d. & n.d. \\
\hline $\begin{array}{l}\text { PF-02- } \\
122\end{array}$ & spindle & 79.28 & 19.83 & 0.11 & 0.42 & 0.08 & 0.28 & n.d. & n.d. & n.d. \\
\hline $\begin{array}{l}\text { PF-02- } \\
129\end{array}$ & spindle & 88.29 & 8.70 & 1.65 & 0.84 & 0.24 & 0.16 & 0.12 & n.d. & n.d. \\
\hline $\begin{array}{l}\text { PF-02- } \\
131\end{array}$ & spindle & 88.57 & 8.95 & 1.70 & 0.41 & 0.07 & 0.19 & 0.08 & 0.03 & n.d. \\
\hline $\begin{array}{l}\text { PF-02- } \\
156\end{array}$ & spindle & 84.17 & 13.09 & 1.10 & 0.37 & 0.15 & 0.88 & 0.24 & n.d. & n.d. \\
\hline $\begin{array}{l}\text { PF-02- } \\
179\end{array}$ & spindle & 91.47 & 6.86 & 0.88 & 0.43 & 0.03 & 0.14 & n.d. & 0.19 & n.d. \\
\hline $\begin{array}{l}\text { PF-02- } \\
182\end{array}$ & spindle & 86.72 & 9.23 & 1.81 & 1.72 & 0.20 & 0.26 & n.d. & 0.06 & n.d. \\
\hline $\begin{array}{l}\text { PF-02- } \\
183\end{array}$ & spindle & 88.63 & 9.67 & 0.63 & 0.22 & 0.10 & 0.67 & 0.08 & n.d. & n.d. \\
\hline $\begin{array}{l}\text { PF-02- } \\
185\end{array}$ & spindle & 84.86 & 13.43 & 1.26 & 0.15 & 0.06 & 0.13 & 0.05 & 0.06 & n.d. \\
\hline $\begin{array}{l}\text { PF-02- } \\
223\end{array}$ & spindle & 90.63 & 5.92 & 1.74 & 0.95 & 0.05 & 0.38 & 0.11 & 0.02 & 0.20 \\
\hline $\begin{array}{l}\text { PF-02- } \\
231\end{array}$ & spindle & 87.98 & 8.25 & 2.06 & 1.03 & 0.10 & 0.14 & n.d. & 0.44 & n.d. \\
\hline $\begin{array}{l}\text { PF-02- } \\
232\end{array}$ & spindle & 82.17 & 14.86 & 1.74 & 0.73 & 0.20 & 0.25 & n.d. & 0.05 & n.d. \\
\hline \multicolumn{11}{|c|}{ Leaded brass } \\
\hline $\begin{array}{l}\text { AA-02- } \\
56\end{array}$ & $\begin{array}{l}\text { casket } \\
\text { ornament }\end{array}$ & 71.20 & 14.75 & 1.75 & 10.90 & 0.32 & 0.42 & 0.20 & 0.02 & 0.44 \\
\hline $\begin{array}{l}\text { BC-01- } \\
81\end{array}$ & earring & 85.07 & 9.78 & 0.12 & 3.70 & 0.13 & 1.05 & 0.15 & n.d. & n.d. \\
\hline $\begin{array}{l}\text { BC-03- } \\
100\end{array}$ & earring & 86.64 & 8.63 & 1.30 & 2.30 & 0.55 & 0.30 & 0.09 & 0.01 & 0.18 \\
\hline $\begin{array}{l}\text { BC-03- } \\
120\end{array}$ & earring & 90.50 & 4.10 & 0.40 & 3.75 & 0.20 & 0.70 & 0.35 & n.d. & n.d. \\
\hline $\begin{array}{l}\text { DV-03- } \\
95\end{array}$ & undetermined & 86.88 & 3.06 & 1.71 & 6.50 & 0.58 & 0.45 & 0.13 & 0.15 & 0.54 \\
\hline
\end{tabular}




\begin{tabular}{|c|c|c|c|c|c|c|c|c|c|c|}
\hline ID & Function & $\mathrm{Cu}$ & $\mathrm{Zn}$ & Sn & $\mathrm{Pb}$ & $\mathrm{Fe}$ & As & $\mathrm{Ag}$ & $\mathrm{Ni}$ & $S b$ \\
\hline $\begin{array}{l}\text { EC-01- } \\
14\end{array}$ & oil lamp stick & 75.84 & 19.69 & 0.07 & 4.19 & 0.07 & 0.12 & n.d. & 0.02 & n.d. \\
\hline $\begin{array}{l}\text { ES-01- } \\
10\end{array}$ & spatula & 89.85 & 2.10 & 1.25 & 6.20 & 0.08 & 0.52 & n.d. & n.d. & n.d. \\
\hline $\begin{array}{l}\text { PF-01- } \\
31\end{array}$ & spindle & 74.97 & 20.58 & 1.10 & 2.20 & 0.14 & 0.52 & 0.18 & 0.03 & 0.28 \\
\hline $\begin{array}{l}\text { PF-01- } \\
38\end{array}$ & spindle & 80.97 & 15.44 & 0.14 & 2.90 & 0.11 & 0.20 & 0.24 & n.d. & n.d. \\
\hline $\begin{array}{l}\text { PF-01- } \\
44\end{array}$ & spindle & 92.88 & 2.24 & 1.14 & 2.85 & 0.04 & 0.25 & 0.21 & n.d. & 0.39 \\
\hline $\begin{array}{l}\text { PF-02- } \\
130\end{array}$ & spindle & 85.54 & 5.83 & 1.93 & 5.74 & 0.40 & 0.36 & 0.20 & n.d. & n.d. \\
\hline $\begin{array}{l}\text { PF-02- } \\
230\end{array}$ & spindle & 91.89 & 3.33 & 1.62 & 2.10 & 0.30 & 0.51 & 0.24 & 0.01 & n.d. \\
\hline \multicolumn{11}{|l|}{ Bronze } \\
\hline $\begin{array}{l}\text { BC-02- } \\
70\end{array}$ & earring & 94.99 & 0.08 & 4.25 & 0.40 & 0.06 & 0.20 & n.d. & 0.02 & n.d. \\
\hline $\begin{array}{l}\text { DV-02- } \\
47\end{array}$ & undetermined & 91.32 & 0.15 & 5.41 & 1.16 & 0.25 & 0.14 & 1.56 & 0.01 & n.d. \\
\hline $\begin{array}{l}\text { DV-02- } \\
64\end{array}$ & undetermined & 90.54 & 0.06 & 9.09 & n.d. & 0.11 & 0.14 & 0.06 & n.d. & n.d. \\
\hline $\begin{array}{l}\text { PF-01- } \\
01\end{array}$ & spindle & 91.34 & 1.54 & 4.63 & 1.10 & 0.20 & 0.61 & 0.17 & n.d. & 0.41 \\
\hline $\begin{array}{l}\text { PF-01- } \\
78\end{array}$ & spindle & 91.94 & 1.94 & 2.93 & 0.79 & 0.20 & 0.26 & 1.94 & n.d. & n.d. \\
\hline \multicolumn{11}{|c|}{ Ternary alloy } \\
\hline $\begin{array}{l}\text { AA-01- } \\
06\end{array}$ & $\begin{array}{l}\text { casket } \\
\text { ornament }\end{array}$ & 83.75 & 10.70 & 2.30 & 1.47 & 0.50 & 0.25 & 0.85 & 0.03 & 0.15 \\
\hline $\begin{array}{l}\text { AA-01- } \\
08\end{array}$ & $\begin{array}{l}\text { casket } \\
\text { ornament }\end{array}$ & 84.40 & 11.00 & 3.00 & 1.05 & 0.15 & 0.17 & 0.10 & 0.03 & 0.10 \\
\hline $\begin{array}{l}\text { AA-01- } \\
18\end{array}$ & $\begin{array}{l}\text { casket } \\
\text { ornament }\end{array}$ & 88.60 & 7.40 & 2.10 & 1.10 & 0.05 & 0.32 & 0.08 & 0.15 & 0.20 \\
\hline $\begin{array}{l}\text { AA-02- } \\
49\end{array}$ & $\begin{array}{l}\text { casket } \\
\text { ornament }\end{array}$ & 87.92 & 7.76 & 3.40 & 0.34 & 0.50 & 0.08 & n.d. & n.d. & n.d. \\
\hline $\begin{array}{l}\text { AA-03- } \\
79\end{array}$ & $\begin{array}{l}\text { casket } \\
\text { ornament }\end{array}$ & 84.52 & 10.39 & 3.30 & 1.40 & 0.17 & 0.20 & n.d. & 0.02 & n.d. \\
\hline
\end{tabular}




\begin{tabular}{|c|c|c|c|c|c|c|c|c|c|c|}
\hline ID & Function & $\mathrm{Cu}$ & $\mathrm{Zn}$ & Sn & $\mathrm{Pb}$ & $\mathrm{Fe}$ & As & $\mathrm{Ag}$ & $\mathrm{Ni}$ & $\mathrm{Sb}$ \\
\hline $\begin{array}{l}\text { AN-01- } \\
35\end{array}$ & ring & 88.52 & 7.48 & 2.64 & 0.50 & 0.05 & 0.55 & 0.08 & 0.01 & 0.17 \\
\hline $\begin{array}{l}\text { BC-01- } \\
09\end{array}$ & earring & 91.40 & 5.60 & 1.95 & 0.57 & 0.06 & 0.15 & 0.10 & n.d. & 0.17 \\
\hline $\begin{array}{l}\text { BC-01- } \\
12\end{array}$ & earring & 89.71 & 5.45 & 3.65 & 0.60 & 0.02 & 0.27 & 0.09 & n.d. & 0.21 \\
\hline $\begin{array}{l}\text { BC-01- } \\
82\end{array}$ & earring & 90.13 & 4.81 & 2.41 & 1.25 & 0.50 & 0.90 & n.d. & n.d. & n.d. \\
\hline $\begin{array}{l}\text { BC-03- } \\
116\end{array}$ & earring & 86.52 & 6.27 & 4.85 & 1.15 & 0.20 & 1.00 & n.d. & 0.01 & n.d. \\
\hline $\begin{array}{l}\text { EC-01- } \\
06\end{array}$ & oil lamp stick & 87.76 & 8.35 & 2.35 & 0.90 & 0.07 & 0.32 & 0.11 & 0.01 & 0.13 \\
\hline $\begin{array}{l}\text { EC-01- } \\
08\end{array}$ & oil lamp stick & 82.46 & 12.62 & 2.75 & 1.25 & 0.05 & 0.12 & 0.40 & n.d. & 0.35 \\
\hline $\begin{array}{l}\text { ES-02- } \\
26\end{array}$ & spatula & 86.74 & 9.39 & 2.30 & 0.81 & 0.04 & 0.32 & 0.13 & n.d. & 0.27 \\
\hline $\begin{array}{l}\text { ES-02- } \\
36\end{array}$ & spatula & 88.22 & 8.05 & 2.50 & 0.58 & 0.25 & 0.26 & 0.14 & n.d. & n.d. \\
\hline $\begin{array}{l}\text { PF-01- } \\
03\end{array}$ & spindle & 89.18 & 6.13 & 2.82 & 0.78 & 0.15 & 0.59 & 0.10 & n.d. & 0.25 \\
\hline $\begin{array}{l}\text { PF-01- } \\
05\end{array}$ & spindle & 91.77 & 4.23 & 2.25 & 0.86 & 0.14 & 0.37 & 0.37 & 0.01 & n.d. \\
\hline $\begin{array}{l}\text { PF-01- } \\
11\end{array}$ & spindle & 86.88 & 11.59 & 0.43 & 0.25 & 0.12 & 0.72 & n.d. & 0.01 & n.d. \\
\hline $\begin{array}{l}\text { PF-01- } \\
30\end{array}$ & spindle & 77.77 & 17.61 & 3.68 & 0.74 & 0.09 & 0.11 & n.d. & n.d. & n.d. \\
\hline $\begin{array}{l}\text { PF-01- } \\
55\end{array}$ & spindle & 87.74 & 7.03 & 3.35 & 1.10 & 0.29 & 0.24 & 0.25 & n.d. & n.d. \\
\hline $\begin{array}{l}\text { PF-01- } \\
99\end{array}$ & spindle & 90.06 & 4.15 & 3.80 & 1.25 & 0.15 & 0.18 & 0.12 & 0.02 & 0.27 \\
\hline $\begin{array}{l}\text { PF-02- } \\
128\end{array}$ & spindle & 84.22 & 10.78 & 2.97 & 1.15 & 0.48 & 0.34 & n.d. & 0.06 & n.d. \\
\hline $\begin{array}{l}\text { PF-02- } \\
161\end{array}$ & spindle & 84.89 & 11.74 & 2.19 & 0.75 & 0.15 & 0.26 & n.d. & 0.02 & n.d. \\
\hline $\begin{array}{l}\text { PF-02- } \\
178\end{array}$ & spindle & 87.33 & 8.06 & 2.90 & 1.18 & 0.15 & 0.30 & n.d. & 0.08 & n.d. \\
\hline
\end{tabular}




\begin{tabular}{|c|c|c|c|c|c|c|c|c|c|c|}
\hline ID & Function & $\mathrm{Cu}$ & $\mathrm{Zn}$ & Sn & $\mathrm{Pb}$ & $\mathrm{Fe}$ & As & $\mathrm{Ag}$ & $\mathrm{Ni}$ & Sb \\
\hline $\begin{array}{l}\text { AA-01- } \\
21\end{array}$ & $\begin{array}{l}\text { casket } \\
\text { ornament }\end{array}$ & 88.10 & 3.70 & 3.05 & 4.00 & 0.50 & 0.37 & 0.09 & 0.02 & 0.17 \\
\hline $\begin{array}{l}\text { AA-02- } \\
39\end{array}$ & $\begin{array}{l}\text { casket } \\
\text { ornament }\end{array}$ & 84.68 & 7.33 & 5.30 & 2.15 & 0.24 & 0.07 & 0.10 & 0.01 & 0.12 \\
\hline $\begin{array}{l}\text { AR-01- } \\
53\end{array}$ & ring & 75.13 & 2.12 & 9.75 & 11.05 & 0.55 & 0.80 & n.d. & 0.05 & 0.55 \\
\hline $\begin{array}{l}\text { BC-01- } \\
86\end{array}$ & earring & 68.74 & 18.08 & 6.25 & 6.30 & 0.09 & 0.30 & 0.21 & 0.03 & n.d. \\
\hline $\begin{array}{l}\text { BC-03- } \\
118\end{array}$ & earring & 83.57 & 10.94 & 2.34 & 2.25 & 0.32 & 0.50 & n.d. & 0.08 & n.d. \\
\hline $\begin{array}{l}\text { DV-01- } \\
31\end{array}$ & undetermined & 82.25 & 9.30 & 4.30 & 3.60 & 0.15 & 0.10 & 0.09 & 0.01 & 0.20 \\
\hline $\begin{array}{l}\text { DV-02- } \\
63\end{array}$ & undetermined & 82.71 & 4.96 & 2.12 & 7.85 & 0.40 & 0.64 & 0.20 & 0.10 & 1.02 \\
\hline $\begin{array}{l}\text { EC-01- } \\
01\end{array}$ & oil lamp stick & 85.62 & 8.73 & 2.12 & 2.75 & 0.25 & 0.12 & 0.14 & n.d. & 0.27 \\
\hline $\begin{array}{l}\text { PF-01- } \\
08\end{array}$ & spindle & 69.34 & 21.27 & 2.33 & 5.90 & 0.17 & 0.77 & 0.15 & 0.07 & n.d. \\
\hline $\begin{array}{l}\text { PF-01- } \\
14\end{array}$ & spindle & 82.98 & 5.71 & 4.00 & 6.60 & 0.51 & 0.20 & n.d. & n.d. & n.d. \\
\hline \multicolumn{11}{|c|}{ Unalloyed copper } \\
\hline $\begin{array}{l}\text { AA-02- } \\
43\end{array}$ & $\begin{array}{l}\text { casket } \\
\text { ornament }\end{array}$ & 99.52 & 0.08 & n.d. & 0.21 & 0.01 & 0.15 & n.d. & 0.03 & n.d. \\
\hline $\begin{array}{l}\text { AA-03- } \\
59\end{array}$ & $\begin{array}{l}\text { casket } \\
\text { ornament }\end{array}$ & 96.45 & 1.90 & 0.26 & 0.65 & 0.07 & 0.16 & 0.15 & 0.06 & 0.30 \\
\hline $\begin{array}{l}\text { AA-03- } \\
66\end{array}$ & $\begin{array}{l}\text { casket } \\
\text { ornament }\end{array}$ & 97.05 & 0.13 & 1.30 & 0.15 & 0.29 & 0.65 & 0.05 & 0.08 & 0.30 \\
\hline $\begin{array}{l}\text { BC-03- } \\
117\end{array}$ & earring & 96.13 & 1.58 & 0.87 & 0.45 & 0.23 & 0.63 & n.d. & 0.11 & n.d. \\
\hline $\begin{array}{l}\text { DV-01- } \\
04\end{array}$ & undetermined & 98.00 & 0.09 & 0.10 & 0.63 & 0.13 & 0.09 & 0.62 & 0.04 & 0.30 \\
\hline $\begin{array}{l}\text { DV-01- } \\
05\end{array}$ & undetermined & 98.92 & 0.10 & n.d. & 0.03 & 0.25 & 0.13 & 0.57 & n.d. & n.d. \\
\hline $\begin{array}{l}\text { DV-01- } \\
36\end{array}$ & undetermined & 98.55 & 0.09 & n.d. & 0.33 & 0.10 & 0.06 & 0.66 & 0.02 & 0.19 \\
\hline $\begin{array}{l}\text { DV-02- } \\
53\end{array}$ & undetermined & 97.70 & 0.10 & 0.95 & 0.80 & 0.02 & 0.14 & 0.11 & 0.02 & 0.16 \\
\hline
\end{tabular}




\begin{tabular}{|c|c|c|c|c|c|c|c|c|c|c|}
\hline ID & Function & $\mathrm{Cu}$ & Zn & Sn & $\mathrm{Pb}$ & $\mathrm{Fe}$ & As & $\mathrm{Ag}$ & $\mathrm{Ni}$ & Sb \\
\hline $\begin{array}{l}\text { DV-02- } \\
54\end{array}$ & undetermined & 96.44 & 1.77 & 0.85 & 0.35 & 0.17 & 0.24 & n.d. & n.d. & 0.18 \\
\hline $\begin{array}{l}\text { DV-02- } \\
79\end{array}$ & undetermined & 98.07 & 0.07 & 0.26 & 0.80 & 0.04 & 0.31 & 0.25 & 0.02 & 0.18 \\
\hline $\begin{array}{l}\text { FIV-01- } \\
12\end{array}$ & buckle & 98.95 & 0.09 & n.d. & 0.64 & 0.05 & 0.04 & 0.09 & 0.01 & 0.13 \\
\hline $\begin{array}{l}\text { PR-02- } \\
13\end{array}$ & nail & 98.55 & 0.25 & 0.07 & 0.66 & 0.23 & 0.20 & n.d. & 0.04 & n.d. \\
\hline \multicolumn{11}{|c|}{ Leaded unalloyed copper } \\
\hline $\begin{array}{l}\text { AR-01- } \\
42\end{array}$ & ring & 96.63 & 0.05 & 0.63 & 2.30 & 0.03 & 0.25 & 0.11 & n.d. & n.d. \\
\hline $\begin{array}{l}\text { DV-01- } \\
19\end{array}$ & undetermined & 96.48 & 0.09 & 0.49 & 2.30 & 0.20 & 0.18 & 0.09 & 0.09 & 0.08 \\
\hline $\begin{array}{l}\text { PR-02- } \\
06\end{array}$ & nail & 93.62 & 0.11 & 0.15 & 4.85 & 0.24 & 0.46 & 0.16 & 0.03 & 0.38 \\
\hline
\end{tabular}

- unalloyed $\mathrm{Cu}: \mathrm{Cu}+<2 \% \mathrm{Sn}$ and $<2 \% \mathrm{Zn}$;

- bronze: $\mathrm{Cu}+>2 \% \mathrm{Sn}$ and $<2 \% \mathrm{Zn}$;

- brass: $\mathrm{Cu}+>2 \% \mathrm{Zn}$ and $<2 \% \mathrm{Sn}$;

- ternary alloy (modern gunmetal): $\mathrm{Cu}+>2 \% \mathrm{Sn}$ and $>2 \% \mathrm{Zn}$;

- Leaded alloys: $>2 \% \mathrm{~Pb}$.

According to the adopted criteria, brass was the most frequent alloy in use at Mértola, being represented by 118 artefacts, 12 of which with more than $2 \mathrm{wt} . \% \mathrm{~Pb}$. Seventeen objects were made of unalloyed $\mathrm{Cu}$, three of which with more than $2 \mathrm{wt} . \% \mathrm{~Pb}$. Ternary alloys are 36,10 of which with more than $2 \mathrm{wt} . \% \mathrm{~Pb}$. Finally, there are six bronzes, none of them with $\mathrm{Pb}$ higher than 2 wt.\% (Fig. 4A). As for minor elements, i.e., $\mathrm{Zn}, \mathrm{Sn}$, and $\mathrm{Pb}$ when $<2 \mathrm{wt} . \%, \mathrm{Fe}, \mathrm{As}, \mathrm{Ag}, \mathrm{Ni}$, and $\mathrm{Sb}$, their content tends to be quite variable, ranging from 0.21 to $3.25 \mathrm{wt} . \%$ in total.

At first glance, the results showed $\mathrm{Zn}$ to be the principal element to have been mixed with $\mathrm{Cu}$ and brass is the main alloy detected (Fig.4 B). At the same time, the data also indicate a seemingly random and unpredictable variability of the major components, i.e., $\mathrm{Zn}, \mathrm{Sn}$ and $\mathrm{Pb}$.

As the addition of each of these elements is known to alter the specific mechanical properties of a metal, a major archaeological question to address would be to find the reasons behind this variability. In this sense, the presence/absence of standardised compositional patterns can shed light not only on production technology aspects, but also on a range of issues related to different features of past societies. In fact, social strategies, technological choices, and economic constraints may have an impact 
on any single phase in the manufacturing process, including mining, smelting, melting, and casting processes, and any decisions made along the production chain, whether conscious or irrational, can directly affect the characteristics of the finished objects.

Based on this assumption, and looking at the overall Mértola data, it is quite clear that the high variability of the main elements found in the objects analysed does not allow clear compositional patterns to be identified. Tools, ornaments, and fragments that do not fall into any of the previous categories seem to be produced seamlessly together with alloys containing variable concentrations of $\mathrm{Zn}, \mathrm{Sn}$ and $\mathrm{Pb}$.

Considering the high technological expertise in metal production reached during the Islamic Iberian Peninsula $[12,14,17]$, it is not at all likely that the metalworkers who produced the objects found in Mértola were unaware of the mechanical properties of the different copper-based alloys to the point of not taking advantage of them. A detailed analysis of each of the alloys identified at Mértola will be presented with the aim of discussing the probable causes of this elemental variability.

\section{Brass artefacts (Cu-Zn)}

Brass is the predominant alloy produced and used at Merrtola. It representsalmost $69 \%$ of the entire assemblage analysed in this work, having been used to produce different types of metals, namely: 37 spindles, 31 earrings, 21 casket ornaments, 13 spatulas, 6 undetermined, 2 rings, and 1 sword sheath. Furthermore, only 12 out of 118 brasses contain more than $2 \%$ of $\mathrm{Pb}$ (Fig. $5 \mathrm{~A}$ ).

Overall, Zn ranges from 2.1 to $22.3 \mathrm{wt}$.\%, showing an average of c. $9.5 \mathrm{wt} . \%$. Only one of the objects analysed (AA-02-29) has a Zn content higher than $22 \mathrm{wt} . \%$, thus falling into the $22-28 \mathrm{wt} . \% \mathrm{Zn}$ range, that is the typical interval for brasses produced with the method of cementation [18-20].

Fig. $5 \mathrm{~B}$ confirms the lack of correlation between $\mathrm{Zn}$ variability and the functionality of the artefacts. In fact, a $\mathrm{Zn}$ content in the range of $10-20 \mathrm{wt}$.\% is known to be responsible for a golden yellow colour in the final alloy, making the latter particularly suitable for ornamental objects. When looking at Mértola's data, though, the 40 artefacts that fall in this range are evenly distributed between tools and ornaments, suggesting that the brightness and colour nature of an alloy was probably not considered relevant in producing finished objects with specific forms and functions.

With only few exceptions, almost all the brasses (105 out of 118) contain Zn between $4 \%$ and $20 \%$ (Fig. $5 \mathrm{C})$, that is the range that Craddock considers as typical for objects produced through the mixing of pristine brass with 22 to $28 \mathrm{wt}$.\% Zn with scrap copper-based alloys with lower Zn content [18].

The overall $\mathrm{Zn}$ content, however, is evidence for the use of scrap metals as raw materials to produce new objects. Further evidence in support of this hypothesis is the moderate and apparently random occurrence of $\mathrm{Zn}$. When a brass is remelted, the alloy progressively loses about $10 \mathrm{wt} . \%$ of its $\mathrm{Zn}$ content, and a 4 to $5 \mathrm{wt}$ \% additional $\mathrm{Zn}$ should be added to compensate the melting losses. Considering, for instance, the recycling of an ancient brass produced via the so-called cementation process and containing $28 \% \mathrm{Zn}$, Zn content may drop to about $25 \mathrm{wt} . \%$ after the first remelting, to $22 \mathrm{wt} . \%$ after a 
further remelting, and so on [21]. This means that the $\mathrm{Zn}$ variability observed in Mértola's brass objects is most likely the result of multiple remelting of scrap metal composed, in turn, of alloys with varying $\mathrm{Zn}$ content.

Another point to highlight is the presence of a small group of brass artefacts containing just over $20 \mathrm{wt} . \%$ $\mathrm{Zn}$. These may be brasses that have undergone two or three remelting cycles or, alternatively, may have been produced by cementation throughout the so-called medieval method [22]. This involves the reaction of zinc vapour with liquid rather than solid copper, at higher temperatures and in open vessels. The use of this method at a temperature of about $1200^{\circ} \mathrm{C}$ makes it possible to produce a brass with about $20 \mathrm{wt} \%$ Zn.

In any case, most brasses from the Almohad neighbourhood of Mārtulah contain a moderate Zn content and an overall low level of impurities. According to Gaudenzi Asinelli et al., this could be suggesting the use of sphalerite $((\mathrm{Zn}, \mathrm{Fe}) \mathrm{S})$ as a zinc ore [16]. In the case of the metals from Mértola, this is a relevant data as sphalerite zinc ores are common in the Iberian Pyrite Belt, a metallogenic province located in the SW Iberian Peninsula [23-25], thus suggesting the use of local minerals.

\section{Unalloyed Cu artefacts}

Pure coppers are relatively scarce, accounting for about $8.8 \%$ of the investigated collection. Of the 15 artefacts in this group, 12 contain less than $2 \mathrm{wt} . \% \mathrm{~Pb}$. From a typological perspective, these Cu-artefacts include three casket ornaments, one ring, one earring, one buckle, seven undetermined objects, and two nails. The only two nails analysed in this paper are both made of copper, although with differences in terms of $\mathrm{Pb}$ content. Due to the small number of artefacts composed of unalloyed copper, the data does not allow however for any further noteworthy comment to be made.

\section{Bronze artefacts (Cu-Sn)}

Binary bronzes consist of only six objects (3.5\% of the entire collection). Similar to the other alloys discussed so far, also bronze was not used to produce a specific type of object. Altogether, one earring, two undetermined objects, and two spindles were made of bronze, i.e., one ornament, two unknown, and two tools in terms of functionality.

The Sn content is highly variable, ranging from 2.93 to 9.09 wt.\% (Fig. 6A). Minor elements like $\mathrm{Zn}, \mathrm{Pb}, \mathrm{Fe}$, $\mathrm{As}, \mathrm{Ni}$, and $\mathrm{Sb}$, range from 0.76 to $5.13 \mathrm{wt}$ \% in total, and no leaded bronzes are present (Fig. 6B). Given their residual occurrence, none of impurities had any significant impact on the mechanical properties of the alloys.

As it is well known, the addition of $\mathrm{Sn}$ to $\mathrm{Cu}$ lowers the melting temperature of $\mathrm{Cu}$, and improves the mechanical properties of the metal, making the alloy physically more resistant to impacts. In this respect, the mechanical effects that the presence of Sn may have on the finished alloy begin to become evident only at Sn concentrations above 3-4\% [26], with the best outcome with Sn \% above 10-15 wt.\%. 
Considering the Sn content found in the bronzes from the Almohad neighbourhood of Mértola, it is quite evident that the addition of fresh Sn during the melting process was not a technological option for the metalworkers that produced these metals. As such, the reduced content of $\mathrm{Sn}$ is a further indication that, at that time, the use of recycled scraps as a raw material, instead of alloying $\mathrm{Cu}$ and $\mathrm{Sn}$ in suitable proportions, was a well-established practice. The reduced amount of $\mathrm{Sn}$ in the finished objects is a consequence of the decrease in concentration that this element experiences as a consequence of the recycling process. Each time a tin-bronze is remelted, Sn gradually decreases through volatilization, leading to the production of objects with less Sn content than those used as scrap. The higher the number of remelting episodes, therefore, the lower the amount of tin in the final alloy [18].

When placed in its historical context, the low concentration of tin in the alloys found in Mértola cannot be considered unexpected [27]. In fact, no tin mines have so far been identified in the South of Portugal with the most likely source of tin at the beginning of the $2^{\text {nd }}$ millennium being located in the Iberian Peninsula northwest, where tin had been exploited since antiquity [28]. However, it is very likely that with the Reconquista underway, these tin mines were no longer accessible to Moors as in the first quarter of the $2^{\text {nd }}$ millennium Iberia northwest was already under the firm control of the Christian kingdoms.

The low concentration of tin in the alloys analysed in this paper could therefore be explained by a shortage of Sn supply due to the interruption of the tin trade to southern Portugal. However, it cannot be underestimated that tin, during the Islamic period, was also used for other craft productions, in particular for pottery glazes [29-35]. Thus, it is also possible that the little available tin, given its scarcity, may have been deliberately restricted to productions of greater social and artistic values such as prestige pottery, rather than for metal objects of daily use.

\section{Ternary alloys artefacts (Zn-Sn-Pb)}

Ternary alloys represent $18.7 \%$ of the entire assemblage, and includes ten spindles, seven casket ornaments, six earrings, three oil lamp sticks, two rings, two spatulas, and two undetermined objects.

All the ternary alloys show very reduced tin concentration, and a variability in composition of both $\mathrm{Zn}$ (from 2 to 13 wt.\%), and $\mathrm{Pb}$ (26 of the 36 ternary objects have less than 2 wt.\% $\mathrm{Pb}$, ranging from 0.3 to 11 wt.\%) (Fig. 7). A clear pattern linking the variability of major elements and the typology of the artefacts is, once again, not detectable as evidenced by Fig. 8.

The composition of ternary alloys appears to be a further argument in favour of a predominantly scrapbased metallurgy in which fresh ores were not added to the melt. Low levels of $\mathrm{Zn}$ and Sn, in particular, confirm the hypothesis already discussed above which regards the use of scrap as raw material to produce new objects as a very common practice of the time.

\section{Impurities}


As for impurities, i.e., elements whose concentration is less than $2 \mathrm{wt} . \%$, they most likely derive from minor metallic elements present in the ore and reduced unintentionally during the smelting process that end up incorporated into the finished objects. Their concentration depends on different factors such as the quantity of impurities in the ore or the smelting technology in use. Attempting to address questions concerning to origin of raw materials through the identification of impurity patterns is a controversial issue in archaeology, although it is quite clear that some chemical characteristics of the ore are still detectable in the finished objects. The point here is that the composition of an ore source is not homogeneous, and impurities can vary even at different locations within the same ore (Fig. 9).

\section{Conclusion}

This research has shown that a variety of different Cu-based alloys were in use in Mértola during the 12th and the first half of the 13th centuries, i.e., during the Islamic period. Tin bronze artefacts are the smallest group, while brass appears to be the preferred alloy to produce objects of daily use. Moreover, bronzes and brasses were further mixed to produce ternary alloys $(\mathrm{Cu}+\mathrm{Sn}+\mathrm{Zn})$. Occasionally, $\mathrm{Pb}$ was randomly added to the different alloys.

The variability of major elements alloyed to $\mathrm{Cu}$, i.e., $\mathrm{Zn}, \mathrm{Sn}$, and $\mathrm{Pb}$, could be due to technological constraints or political restrictions or to the combination of both factors.

On the other hand, data suggest that objects were not produced with well-defined and predetermined composition. In fact, the XRF analysis clearly revealed that no link can be found between the functions or the forms of the artefacts and their composition as similar objects were produced with different alloys, and vice versa, objects with distinct forms and functions were made of alloys with very similar mechanical properties. This data could indicate that metalworker that produced the objects found in the Almohad quarter of Mértola apparently did not possess advanced technical skills or they were not particularly concerned with the final alloy composition of the artefacts, and/or even if they were aware of the advantages linked to the different chemical compositions of the alloys, they chose not to take advantage of this knowledge.

However, it is very likely that political barriers and economic constraints of the time could have deeply influenced the technological options that metalworkers took along the production chain. In this respect, mention has been already made of the great instability experienced in southern al-Andalus during the 12th and the first half of the 13th centuries, characterized by periods of strong political fragmentation, i.e., with the formation of the so-called Taifas, and periods of reunification, particularly under the Almoravids and Almohads dynasties. Furthermore, since the beginning of the 2 nd millennium, an increasing intensification of pressure on Islamic territories by Christian forces lead to the conquering South of Portugal finally achieved in the mid-13th century. This climate of political instability is very likely to have had a negative effect on the ore minerals trade, with Islamic communities experiencing ever increasing difficulties in the access to ore mineral resources located in territories they did not control. This is especially true for tin, which unlike zinc ores were not available locally and was also used for other 
types of production, such as glazed pottery. As a result, metal technology was affected by the widespread political insecurity in al-Andalus at the time, and local craftsmen were forced to adapt their production to circumstances beyond their control and to use as raw material local ores or scrap metals they had easier access to.

\section{Declarations}

\section{Acknowledgments}

The authors would like to acknowledge Rute Fortuna, from the Campo Arqueológico de Mértola for the archaeological laboratory treatment of the metals. CB also thanks Dr Lino Mioni (Indiana University Bloomington, US) and Mr Vasco Rossi (Zocca, Italy).

\section{Authors' contributions}

$\mathrm{CB}$ : conceptualisation, methodology, XRF analysis, data collection and interpretation, and writing-original draft preparation. RB: data interpretation and writing editing. MB: XRF analysis, data collection and writing editing, JM: writing editing. SGM and LR: archaeological investigation and writing editing. NS: writing editing. All authors have read and agreed to the published version of the manuscript.

\section{Funding}

The study was carried out within the project DE RE METALLICA - DEfining and REdiscovering MEtallurgy and Trade in AL-Andaluz (VII-XIII century AD): Leaping into Innovative Comprehensive Archaeometric Approaches. Analyses were carried out with equipment from the HERCULES Laboratory (University of Évora, Portugal) and funded by FCT (UIDB/04449/2020). Funding for fee payment was made available by the H2020-MSCA-ITN-EJD ED-ARCHMAT Project funded by the H2020-MSCA-ITN-EJD under GA n. 766311.

\section{Availability of data and materials}

The datasets used and/or analysed during the current study are available from the corresponding author on reasonable request.

\section{Competing interests}

The authors declare that they have no competing interests.

\section{Author details}

${ }^{1}$ HERCULES Laboratory, University of Évora, Portugal. ${ }^{2}$ City University of Macau Chair in Sustainable Heritage, University of Évora, Portugal. ${ }^{3}$ University of Évora, Portugal. ${ }^{4}$ CEAACP - Centro de Estudos em Arqueologia, Artes e Ciências do Património. ${ }^{5}$ Universidade Católica Portuguesa, School of Arts, Research Centre for the Science and Technology of the Arts, Porto, Portugal. ${ }^{6}$ Municipality of Mértola, Portugal. 


\section{References}

1. Al-Hassan AY, Hill DR. Islamic technology: an illustrated history, Cambridge: Cambridge University Press, 1986.

2. Kraemer JL. Humanism in the Renaissance of Islam, Leiden: Brill Publishers, 1992.

3. Al-Hassan AY. Transfer of Islamic Science to the West. Manchester: Foundation for Science Technology and Civilisation, 2006.

4. Corfis IA. Three Cultures, One World. In: Corfis IA, editor. Al-Andalus, Sepharad and Medieval Iberia: Cultural Contact and Diffusion. Leiden: Brill Publishers, 2009, p. iii-xiv.

5. Falagas ME, Zarkadoulia EA, Samonis G. Arab science in the golden age (750-1258 C.E.) and today. The FASEB Journal. 2006, 20:1581-6.

6. Renima A, Tiliouine H, Estes RJ. The Islamic Golden Age: A Story of the Triumph of the Islamic Civilization. In: Tiliouine H, Estes RJ, editors. The State of Social Progress of Islamic Societies. Springer International Publishing, 2016. p. 25-52.

7. Macias S. Mértola O último porto do Mediterrâneo. Mértola: Campo Arqueológico de Mértola, 2005.

8. Gómez Mártinez S. New Perspectives in the Study of Al-Andalus Ceramics, Mértola (Portugal) and the Mediterranean Maritime Routes in the Islamic Period. Al-Masaq. 2009. https://doi.org/10.1080/09503110802704437.

9. Gómez-Mártinez S, Rafael L, Macias S. Habitat e utensílios na Mértola almóada. Cuadernos de Madinat al-Zahra. 2010, 7:175-195.

10. Gener M, Montero-Ruiz I, Murillo-Barroso M, Manzano E, Vallejo A. Lead provenance study in medieval metallic materials from Madinat al-Zahra (Medina Azahara, Córdoba). J Archaeol Sci. 2014. https://doi.org/10.1016/j.jas.2014.01.029.

11. Ponting M. From Damascus to Denia: Scientific Analysis of Three Groups of Fatimid Period Metalwork. Historical Metallurgy. 2003, 37(2):85-105.

12. Azuar Ruíz R. Los bronces islámicos de Denia (s.V HG/XI d.C.). Alicante: MARQ - Museo Arqueológico de Alicante, 2012.

13. Gonçalves MJ, P Valério, MF Araújo, Um molde islâmico de um arrabalde da cidade de Silves. XELB. 2008, 8(2):169-176.

14. Bottaini C, Mirão J, Candeias A., Catarino H, Silva RJ, Brunetti A. Elemental characterisation of a collection of metallic oil lamps from South-Western al-Andalus using EDXRF and Monte Carlo simulation, Eur Phys J Plus. 2019. https://doi.org/10.1140/epjp/i2019-12894-4.

15. Ingelbrecht C, Adriaens A, Maier E. Certification of arsenic, lead, zinc and tin (mass fractions) in five copper alloys, BCR 691, Report EUR 19778/1, Directorate General for Research, 2001.

16. Gaudenzi Asinelli M, Martinón-Torres M. Copper-alloy use in a Tyrrhenian medieval town: The case of Leopoli-Cencelle (Italy), J Archaeol Sci Rep. 2016. https://doi.org/10.1016/j.jasrep.2015.09.023. 
17. Hernández Sánchez F. Nueva aproximación al estudio de varias piezas suntuarias islâmicas metálicas del Museo Arqueológico Nacional. Boletín del Museo Arqueológico Nacional. 2017, 36:261-276.

18. Craddock P. The composition of the copper alloys used by the Greek, Etruscan and Roman civilizations, J Archaeol Sci. 1978. https://doi.org/10.1016/0305-4403(78)90015-8.

19. Dungworth D. Roman Copper Alloys: Analysis of Artefacts from Northern Britain, J Archaeol Sci. 1997. https://doi.org/10.1006/jasc.1996.0169.

20. Newbury BD, Notis M, Newbury DE. Revisiting the zinc composition limit of cementation brass. Historical Metallurgy. 2005, 39:75-81.

21. Ponting M. East Meets West in Post-Classical Bet She'an: The Archaeometallurgy of Culture Change, J Archaeol Sci. 1999. https://doi.org/10.1006/jasc.1998.0373.

22. Rehren T."The same... ... but different": A juxtaposition of Roman and Medieval brass making in Central Europe. In: Young SMM, Pollard AM, Budd P, Ixer RA, editors. Metals in Antiquity. Oxford: BAR International Series 792, 1999. p. 252-256.

23. Sáez R, Almodóvar GR, Pascual E. Geological constraints on massive sulphide genesis in the Iberian Pyrite Belt. Ore Geol Rev. 1996. https://doi.org/10.1016/S0169-1368(96)00012-1.

24. Oliveira DPS de, Mtos JX, Rosa CJP, Rosa DRN, Figueiredo MO, Silva TP, Guimarães F, Carvalho JRS, Pinto AMM, Relvas JRMS, Reiser FKM. The Lagoa Salgada Orebody, Iberian Pyrite Belt, Portugal. Econ Geol. 2011. https://doi.org/10.2113/econgeo.106.7.1111.

25. Almodóvar GR, Yesares L, Sáez R, Toscano M, González F, Pons JM. Massive Sulfide Ores in the Iberian Pyrite Belt: Mineralogical and Textural Evolution. Minerals. 2019. https://doi.org/10.3390/min9110653.

26. Philip G. Tin, Arsenic, Lead: Alloying Practices in Syria-Palestine around 2000 B.C. Levant. 1991. https://doi.org/10.1179/lev.1991.23.1.93.

27. Craddock P. The copper alloys of the Medieval Islamic world-inheritors of the Classical tradition, World Archaeol. 1979. https://doi.org/10.1080/00438243.1979.9979750.

28. Comendador Rey B, Meunier E, Figueiredo E, Lackinger A, Fonte J, Fernández Fernández C, Lima A, Mirão J, Silva RJC. Northwestern Iberian Tin Mining from Bronze Age to Modern Times: an overview. In: Newman P, editor. The Tinworking Landscape of Dartmoor in a European Context - Prehistory to 20th Century: Papers Presented at a Conference in Tavistock, Devon, 6-11 May 2016 to Celebrate the 25th Anniversary of the DTRG. Woolwell, Plymouth: Dartmoor Tinworking Research Group, 2016. p. 133-153.

29. Mason RB, Tite MS. The beginnings of tin-opacification of pottery glazes. Archaeometry. 1997. https://doi.org/10.1111/j.1475-4754.1997.tb00789.x.

30. Molera J, Vendrell-Saz M. Chemical and Textural Characterization of Tin Glazes in Islamic Ceramics from Eastern Spain. J Archaeol Sci. 2001. https://doi.org/10.1006/jasc.2000.0606.

31. Molera J, Coll J, Pradell T. Manganese brown decorations in 10th to 18th century Spain tin glazed ceramics. Appl Clay Sci. 2013. https://doi.org/10.1016/j.clay.2013.05.018. 
32. Tite $M$, Watson $\mathrm{O}$, Pradell $\mathrm{T}$, Matin M, Molina G, Domoney K, Bouquillon A. Revisiting the beginnings of tin-opacified Islamic glazes. J Archaeol Sci. 2015. https://doi.org/10.1016/j.jas.2015.02.005.

33. Tite M, Pradell T, Shortland A. Discovery, production and use of tin-based opacifiers in glasses, enamels and glazes from the Late Iron Age onwards: a reassessment. Archaoemetry. 2017. https://doi.org/10.1111/j.1475-4754.2007.00339.x.

34. Matin $M$, Tite $M$, Watson 0 . On the origins of tin-opacified ceramic glazes: New evidence from early Islamic Egypt, the Levant, Mesopotamia, Iran, and Central Asia. J Archaeol Sci. 2018. https://doi.org/10.1016/j.jas.2018.06.011.

35. Matin M. Tin-based opacifiers in archaeological glass and ceramic glazes: a review and new perspectives. Archaeol Anthrop Sci. 2019. https://doi.org/10.1007/s12520-018-0735-2.

\section{Figures}




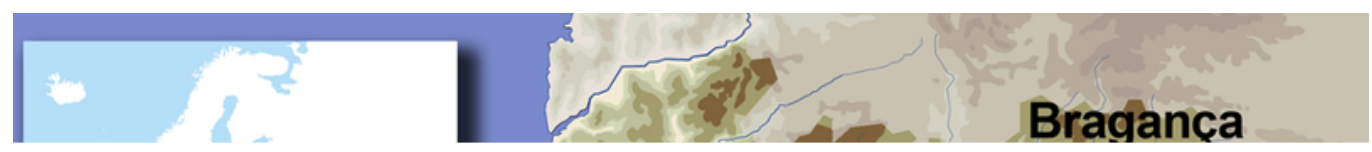

Figure 1

Location of Mértola (South of Portugal). 
Figure 2

The Almohad neighbourhood. Aerial view (A), and detail of an archaeological area of the excavation (B).

$\left.\left.||^{2}\right|^{3} p^{4}\right|^{5} /\left.\left.\right|^{6}\right|^{7} \mathbf{Q}^{8}-\|^{11}$

Figure 3

A selected group of metals analysed in this paper. 


\section{Figure 4}

Histogram showing the distribution of artefacts within the different kind of alloys found at Mértola (A). Bivariate plot displaying $\mathrm{Sn}$ and $\mathrm{Zn}$ values for the objects analysed in this paper (B)

\section{Figure 5}

Composition of the different types of metals (A), plot displaying the lack of any correlation between the concentration of $\mathrm{Zn}$ and the functionality of artefacts (B), and frequency histogram of $\mathrm{Zn}$ in the brasses(C)

\section{Figure 6}

Frequency of bronze artefacts showing the variability of $\mathrm{Sn}(\mathrm{A})$, and $\mathrm{Pb}(\mathrm{B})$, within the bronze artefacts.

\section{Figure 7}

Variability of $\mathrm{Zn}(\mathrm{A}), \mathrm{Sn}(\mathrm{B})$, and $\mathrm{Pb}(\mathrm{C})$ within the ternary alloy artefacts.

\section{Figure 8}

Ternary alloys showing the lack of correlation between the variability of $\mathrm{Zn}, \mathrm{Sn}$, and $\mathrm{Pb}$ concentration in ternary alloys and the function of the artefacts (red dots: undetermined, green dots: tools, black dots: ornament). 


\section{Figure 9}

SEM-EDS mapping of the nail PR-02-06, showing Pb-rich inclusions in a pure copper alloy 